\section{Years of Advancing the Standard of Cancer Care}

\author{
Robert W. Carlson, MD
}

This year's NCCN Annual Conference: Advancing the Standard of Cancer Care, hosted at The Diplomat Hotel in Hollywood, Florida, March 12 through 14, marked a special milestone for the organization. It was not only the 20th Annual Conference, but also a celebration of NCCN's 20th year working to improve the quality, effectiveness, and efficiency of cancer care so that patients can lead better lives. We celebrated this milestone with an agenda comprising a multitude of experts throughout the oncology landscape, as well as exciting new developments in more than 15 cancer types and emerging therapies. This year's conference was a true testament to the magnitude of impact NCCN and its Member Institutions have had on the treatment of cancer care over the past 2 decades, and we present these Highlights as a synopsis of the proceedings in this special issue of JNCCN-Journal of the National Comprehensive Cancer Network.

The 3 days of the NCCN 20th Annual Conference included a variety of educational activities. For example, "Value-Based Decision-Making at the Bedside" used a roundtable format to explore tools such as clinical guidelines and pathways and the impact such tools have on clinician and patient decision-making at the bedside. Moderated by Clifford Goodman, PhD, The Lewin Group, the roundtable included panelists Peter B. Bach, MD, MAPP, Memorial Sloan Kettering Cancer Center; Stephen B. Edge, MD, Baptist Cancer Center; Linda House, RN, BSN, MSM, Cancer Support Community; Jennifer Malin, MD, PhD, WellPoint/Anthem; and James L. Mohler, MD, Roswell Park Cancer Institute.

Another roundtable, "What Are the Characteristics of an Optimal Clinical Practice Guideline?" was moderated by F. Marc Stewart, MD, Fred Hutchinson Cancer Research Center/Seattle Cancer Care Alliance. This roundtable covered the process through which the NCCN Guidelines are created, with panelists that included David S. Ettinger, MD, The Sidney Kimmel Comprehensive Cancer Center at Johns Hopkins; Michael Kuettel, MD, MBA, PhD, Roswell Park Cancer Institute; Jennifer Malin, MD, PhD, WellPoint/Anthem; Joan S. McClure, MS, NCCN; Mary Lou Smith, JD, MBA, Research Advocacy Network; and Andrew D. Zelenetz, MD, $\mathrm{PhD}$, Memorial Sloan Kettering Cancer Center.

In addition to expert panels, this year's conference featured a new, customizable format in which attendees could select track sessions. In these presentations, experts outlined some of the latest treatment advances for a variety of tumor types, including bladder, breast, colorectal, esophageal, non-small cell lung, kidney, and prostate cancers, as well as melanoma and hematologic malignancies.

The newest addition to the library of NCCN Guidelines-those for Smoking Cessation-were also introduced at the conference. The NCCN Guidelines for Smoking Cessation provide detailed pharmacotherapy and behavioral therapy recommendations for people with cancer who smoke.

And, as I noted in my Keynote Address at the conference (see page 629), we also have a number of transformative initiatives in the works. For the NCCN Guidelines, these include collaboration with IBM to create digital-based guidelines that can be processed and implemented across a wide range of clinical support systems; enhanced description of efficacy, safety, data quality, and cost; and resource stratification.

As the NCCN Guidelines change and improve, NCCN also continues to develop and improve other projects. For example, derived from the NCCN Guidelines,

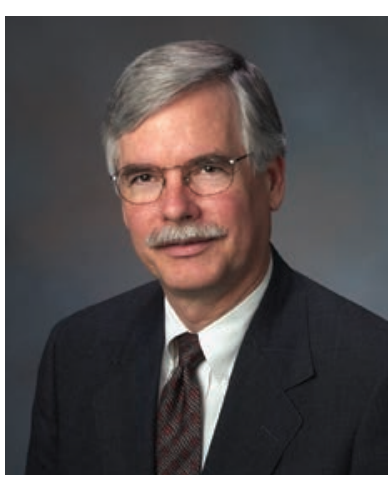

Robert W. Carlson, MD

Robert W. Carlson, MD, joined NCCN as CEO in January 2013 after numerous leadership positions, including chair of the NCCN Breast Cancer Panel and several NCCN Task Forces. He has also been a member of the NCCN Breast Cancer Risk Reduction Panel, Breast Cancer Disease-Specific Executive Committee, CME Advisory Committee, Guidelines Steering Committee, and Board of Directors. Previously, Dr. Carlson was Professor of Medicine in the Division of Oncology and Stanford Medical Informatics at Stanford University Medical Center. Dr. Carlson received his medical degree from Stanford University Medical School and completed his internship and junior residency in internal medicine at Barnes Hospital Group in St. Louis. He returned to Stanford for his senior residency and postdoctoral fellowship in medical oncology. Dr. Carlson is board certified in internal medicine and holds a subspecialty certification in medical oncology. Dr. Carlson is a member of several medical societies. He lectures extensively and has authored or co-authored over 130 articles, abstracts, and book chapters. He is also Associate Editor of Oncology.

The ideas and viewpoints expressed in this editorial are those of the author and do not necessarily represent any policy, position, or program of NCCN. 
the NCCN Drugs \& Biologics Compendium (NCCN Compendium) the NCCN Chemotherapy Order Templates, and the NCCN Biomarkers Compendium are clinical resources formatted for ease-of-use for multiple stakeholders. The NCCN Compendium is officially recognized by the Centers for Medicare \& Medicaid Services (CMS), Cigna, and UnitedHealthcare as a mandated reference in the creation of coverage policy and coverage decisions. Further, NCCN collaborates with Health Information Technology organizations to ensure that the NCCN clinical resources are contained in these tools and are available to clinicians, payers, and other health care professionals.

To enhance its commitment to patients, NCCN published the first NCCN Guidelines for Patients in 2010, providing a patient-friendly translation of the NCCN Guidelines for Breast Cancer. Since then, the library of NCCN Guidelines for Patients has grown to include 14 disease types that are available free-of-charge on NCCN.org/ patients. NCCN Guidelines for Patients are made possible by charitable donations to the NCCN Foundation.

In recognition of the essential role of research in advancing cancer therapies in order to improve the quality of life for patients and reduce cancer-related deaths, the NCCN Oncology Research Program (ORP) was established in 1999. The NCCN ORP supports oncology preclinical, translational, and clinical research at NCCN Member Institutions. To date, more than $\$ 50$ million in research grants have been secured and distributed to investigators from NCCN. It is NCCN's vision to have the NCCN ORP become a Center of Excellence in the United States for providing outstanding research and research services.

NCCN has also enriched an already well-established education program with an online learning management platform, providing oncology clinicians with expanded and easy-to-access continuing education opportunities based on NCCN content. Each year, more than $175 \mathrm{NCCN}$ continuing education programs are conducted for medical professionals around the world. Moreover, NCCN offers a certificate program-the NCCN International Educational Activities Program-that recognizes physicians outside the United States for completion of NCCN educational content hours.

NCCN also recently announced a collaboration with Foundation for Excellence and Quality in Oncology (ECO) to publish Spanish translations of NCCN Guidelines and regional adaptions for Spain, including breast, colorectal, non-small cell lung, and prostate cancers and melanoma.

NCCN continues our initiative with McKesson Specialty Health to develop value-based pathways for the treatment of common cancers. These clinical practice guidelines are jointly developed by McKesson Specialty Health, The US Oncology Network, and NCCN to consider efficacy, toxicity, and cost when evaluating treatment options in radiation oncology, and are based on the NCCN Guidelines.

These are just a few of the programs and initiatives in the works and on the horizon. Through these programs and initiatives, NCCN aspires to be the world leader in defining and advancing high-quality, high-value cancer care. But none of that is possible without the contributions and support of our Member Institutions and the larger oncology community. We believe that JNCCN readers, whether you are from an NCCN Member Institution or not, also contribute to improving the quality of cancer care. We embrace this collaboration and partnership, and look forward to working with you now and in the future.

Your support of NCCN and our programs is invaluable, and I hope that you will continue to participate in the NCCN Annual Conference (March 31-April 2, 2016) and other NCCN activities and programs. Please visit NCCN.org for more information and to access programs such as the NCCN Guidelines and to watch, and participate in, NCCN's continuing evolution. 